\title{
KULTURSPEZIFISCHE ELEMENTE IN DER MAZEDONISCH- DEUTSCHEN ÜBERSETZUNG VON BLAŽE KONESKIS ERZÄHLUNGEN „LJUBOV“ UND „PESNA“
}

\section{KULTURNOSPECIFIČNI IZRAZI U MAKEDONSKO-NJEMAČKOM PRIJEVODU PRIPOVIJESTI BLAŽE KONESKOG „LJUBOV“I „PESNA“}

\author{
Emilija Bojkovska \\ Philologische Fakultät Blaže Koneski, Skopje, Nordmazedonien \\ Filološki fakultet Blaže Koneski, Skopje, Sjeverna Makedonija
}

Abstract

Im Beitrag wird auf die Darstellung des Kulturbegriffs, der kulturellen Kompetenz des Übersetzers / der Übersetzerin und der translatorischen Verfahren für die kulturspezifischen Elemente anhand von Blaže Koneskis Erzählungen "Һубов / Ljubov" und „Песна / Pesna“" in mazedonischer Sprache sowie deren deutschen Übersetzungen „Liebe“ und „Petres Lied“ die translatorische Übertragung von Sinngehalt (Inhalt) und Stil (Ausdruck) untersucht. Folgende kulturspezifische Elemente werden behandelt: maz. Песна / Pesna > dt. Petres Lied, maz. paкијa / rakija $>$ dt. Rakija, maz. Богоројц̧а / Bogorojca > dt. Mariä Hilmmelfahrt, maz. opo / oro $>\mathrm{dt}$. Kolo und maz. чардак / с̌ардак > dt. Maisscheune. Die Untersuchung umfasst a) die Bestimmung des kulturellen Subsystems, b) die semantische Zuordnung der kulturspezifischen Termini, c) die formale und die referenzielle Übereinstimmung zwischen Original und Übersetzung (z. B. Expansion, Reduktion) sowie d) den translatorischen Umgang mit der Kulturspezifik (Verfremdung oder Einbürgerung). Die Autorin kommentiert und argumentiert die übersetzerischen Lösungen und schlägt teilweise eigene Übersetzungsvorschläge vor.

\section{Einführung}

Sažetak

U članku se analizira prevođenje pripovijesti "Һуббов / Ljubov“ i „Песна / Pesna“ makedonskog pisca Blaže Koneskog sa makedonskog na njemački jezik "Liebe“ i "Petres Lied“ fokusirajući se na kulturnospecifične izraze. Autorica najprije definira pojam kulture i kulturne kompetencije prevoditelja / prevoditeljie i opredjeli prevodilačke strategije kulturnospecifičnih izraza u kontekstu adekvatnog prenošenja sadržaja i stila. Analiziraju se slijedeći kulturnospecifični izrazi: mak. Песна / Pesna > njem. Petres Lied, mak. paкuja/rakija > njem. Rakija, mak. Богоројц̧а / Bogorojca > njem. Mariä Hilmmelfahrt, mak. opo / oro > njem. Kolo i mak. uapdak / čapdak $>$ njem. Maisscheune. Istraživanje obuhvaća a) određivanje kulturnog subsistema, b) semantičku klasifikaciju kulturnospecifičnih izraza, c) formalnu i referencijalnu usklađenost između originala i prijevoda (npr. ekspanzija ili redukcija) i d) prevoditeljsku strategiju kulturno specifičnih izraza (otuđivanje ili naturalizacija). Autorica komentira i argumentira prevoditeljska rješenja te predlaže svoja vlastita prijevodna rješenja. 
„Die Tätigkeit des Übersetzers besteht seit Urzeiten als Notwendigkeit, zwischen verschiedenen Kulturen und Zivilisationen zu vermitteln und fachliche, literarische und religiöse Inhalte zu übermitteln." /1/ Untersuchungsgegenstand der vorliegenden Arbeit sind kulturspezifische Elemente in Blaže Koneskis Erzählungen „Љубов / Ljubov“ und „Песна / Pesna“" sowie in deren deutschen Übersetzungen „Liebe“ und „Petres Lied“ im Hinblick auf das angewandte translatorische Verfahren zur Übertragung von Sinngehalt (Inhalt) und Stil (Ausdruck). Im Vordergrund steht die Frage, mit welchen sprachlichen Mitteln die kulturspezifischen Elemente aus dem mazedonischen Original in die Zielsprache übertragen wurden. Die Analyse des Korpus folgt auf die Darstellung des Kulturbegriffs, der kulturellen Kompetenz des Übersetzers / der Übersetzerin und der translatorischen Verfahren für die kulturspezifischen Elemente.

Die kulturelle Ebene ist von der rein sprachlichen $\mathrm{zu}$ trennen. Die sprachliche Dimension kommt bei Koller /2/ innerhalb der konnotativen und der formal-ästhetischen Äquivalenz vor. Es handelt sich z. B. um die Verwendung spezifischer Ausdrücke (etwa von Turzismen) als Gattungsnamen für Naturerscheinungen, die nicht zum Untersuchungsgegenstand des vorliegenden Beitrags gehören.

\section{Kultur und kulturspezifische Elemente}

Der Kulturbegriff wird im Folgenden im Zusammenhang mit den translatorischen Verfahren für kulturspezifische Elemente erörtert. Die Fülle der Definitonen von Kultur und Kulturspezifischem zeugt von der Komplexität dieses Phänomens, das hier nur bei einigen $\mathrm{Au}$ toren exemplarisch dargestellt werden kann. Die kulturspezifischen Elemente werden in der Fachliteratur teilweise auch Realia/Realien genannt, wobei Uneinigkeit nicht nur über den Terminus, sondern auch über die Begriffsbestimmung besteht (s. u.).

Newmark /3/ versteht unter Kultur "the way of life and its manifestations that are peculiar to a community that uses a particular language as its means of expression". Er unterscheidet zwischen Universalsprache (universal language),
Kultursprache (cultural language) und persönlicher Sprache (personal language). Bei Bezeichnungen für bestimmte Vorgänge/Tätigkeiten wie 'live', 'die', 'swim' sowie bei Artefakten wie 'mirror' und 'table' gebe es keine übersetzerischen Schwierigkeiten. Demgegenüber seien 'monsoon', 'steppe' 'dacha' 'tagliatelle' Kulturwörter, die übersetzerische Schwierigkeiten bereiten, es sei denn, es gäbe eine kulturelle Überschneidung zwischen Ausgangs- und Zielsprache sowie unter den Lesern. Universalwörter wie 'breakfast','embrace' und 'pile' erfüllen häufig eine universelle Funktion, trügen jedoch nicht zur kulturellen Beschreibung des Referenten bei. Wenn man sich jedoch in einer persönlichen Art und Weise ausdrücken würde, z. B. 'his "underlife" (persönliche Eigenschaften und Privatleben) is evident in that poem','he's a monologger (er beendet nie den Satz)', dann handle sich um einen Idiolekt, was in der Regel ebenfalls $\mathrm{zu}$ übersetzerischen Schwierigkeiten führe. Newman teilt die Kulturwörter in folgende Subklassen ein: Umwelt (Flora, Fauna, Winde usw.), materielle Kultur Artefakte (Lebensmittel, Kleidung, Häuser und Städte, Transportmittel), soziale Kultur (Arbeit und Freizeit), Orgsanisationen, Gebräuche usw. Er betrachtet die Sprache nicht "as a component or feature of culture" /4/. Sie sei vielmehr "Ausdruck von Kultur und nicht [...] deren Bestandteil oder Merkmal" /5/.

Kupsch-Losereit /6/ sieht „Kultur [...] als Komplex von sozialen Handlungen (Kunst, Religion, Wissenschaft, Ethik), Bedeutungen und Vorstellungen [an], verstanden als ein System gemeinsamer Formen und Symbole“. Für Mudersbach /7/ ist Kultur die Gesamtheit einzelner Teilbereiche, nämlich einzelner Kultursysteme. Ein Kultursystem ist „eine Konvention, die auf einen Lebensbereich bezogen ist" /8/. Eine Konvention im Hinblick auf einen Lebensbereich ist „eine Festlegung von Handlungsabläufen, zusätzlich mit Rollen von Gegenständen und Personen" $/ \mathbf{9} /$.

Im Zusammenhang mit dem Entsprechungstyp Eins-zu-Null innerhalb der denotativen Äquivalenz untersucht Koller /10/ die vorläufigen Lücken bei „Realia-Bezeichnungen (sog. landeskonventionellen, in einem weiteren Sinne: kulturspezifischen Elementen), d. h. Ausdrücken und 
Namen für Sachverhalte politischer, institutioneller, sozio-kultureller, geographischer Art, die spezifisch sind für bestimmte Länder". Daraus geht hervor, dass Eigennamen zu den Realia gehören. Eine Aufgabe des Übersetzers ist es, diese Lücken zu schließen (s. Pkt. 4.).

Albrecht /11/ betrachtet Gegenstände und Sachverhalte in der außersprachlichen Wirklichkeit, die bei der Übersetzung Schwierigkeiten bereiten können. Er unterscheidet vier Typen der Realia bzw. Kulturspezifika: 1) Naturgegenstände: Tiere, Pflanzen, Landschaftsformen, die nur in bestimmten Gegenständen vorkommen und bei denen sich ie Frage aufdrängt, ob sie allgemein bekannt sind (Löwe, Tiger, Giraffe, Taiga, Geest); 2) Artefakte (für bestimmte Kulturen charakteristische Gegenstände): Spaghetti, Tortellini, Baguette; gnocchi, Hefeweizen; 3) Landes- oder kulturspezifische Institutionen: public school, Mahlzeit, Wie geht's; (maz.anepumuв/aperitiv); 4) Kollektive Einstellungen zu Gegenständen und Sachverhalten: Gans ,dumm'. Nach Albrecht ist der Terminus Realia umfassender, da er Naturgegenstände einschließt. Realia sind nach Reiß /12/ Gegenstände und Einrichtungen, Sitten und Gebräuche, die nur im Land der Ausgangssprache bekannt sind. Markstein /13/ fügt „der gängigen, konkreten Definition der Realie als Element des Alltags, der Geschichte, der Kultur, der Politik, u. drgl. eines bestimmten Volkes, Landes, Ortes, die keine Entsprechung bei anderen Völkern, anderen Ländern, an anderen Orten hat" folgende Feststellung: „Die Realien sind Identitätsträger eines nationalen/ethnischen Gebildes, einer nationalen/ethnischen Kultur - im weiteren Sinne und werden einem Land, einer Region, einem Erdteil zugeordnet". Sogar Toponyme können Realia werden, z. B. Ausschwitz, Hiroschima. Naturerscheinungen gehören hingegen nicht $\mathrm{zu}$ den Realien, die nur etwas vom Menschn Erschaffenes oder Ersonnenes umfassen /14/. Kutz /15/ unterscheidet zwischen Realien (Teilen der ausgangskulturellen Wirklichkeit), Realienabbildern (den kognitiven Repräsentationen dieser Wirklichkeit im Bewusstesein der
Vertreter der Ausgangskultur) und Realienbenennungen (der sprachlichen Äußerungen der Realienabbildern). Der gemeinsame Nenner dieser Definitionen ist, dass sich die Kultur auf das vom Menschen Geschaffene sowie auf kollektive Verhaltensweisen und Einstellungen bezieht. Keine Einigkeit besteht hingegen darüber, ob Eigennamen und Gattungsbezeichnungen für Naturgegenstände zu den kulturspezifischen Elementen gehören. $\mathrm{Zu}$ den Schwierigkeiten der Abgrenzung von Eigennamen gegen Gattungsnamen vgl. Wurm/16/ und Tuovinen /17/.

Im Folgenden werden aus der Überzeugung heraus, dass unverändert aus dem Original übernommene Eigennamen den Leser in das zielkulturelle Umfeld versetzen, bei der Korpusanalyse auch diese berücksichtigt. Obwohl natürlich nicht die Kulturphänomene selbst, sondern deren Bezeichnungen ins Translat übertragen werden, wird im Folgenden unter den Termini kulturspezifisches Element und Realie sowohl das Phänomen wie auch dessen Bezeichnung verstanden.

\section{Kulturelle Kompetenz des Transla- tors / der Translatorin}

Für eine erfolgreiche translatorische Tätigkeit ist die kulturelle Kompetenz des Translators / der Translatorin eine conditio sine qua non. Zu Recht hebt Kučiš /18/ hervor: „Im 21. Jahrhundert werden Übersetzer zu Kommunikationsexperten und Vermittlern im internationalen Bereich mit dem Status eines Fachmanns für transkulturelle Kommunikation." Von Wotjak /19/ ausgehend unterscheidet Kupsch-Losereit /20/ neben 1) sprachkulturspezifischem, 2) kommunikativ-prozeduralem und 3) textuellem Wissen auch noch 4) prognostische Annahmen des Translators hinsichtlich der Verstehensvoraussetzungen und Erwartungen der Zieltextleser sowie der Projektion und Konstruktion des Fremden. 1) Das sprachkulturspezifische Wissen bezieht sich auf das kulturell geprägte Sach-/Denotatswissen, zu dem das Wissen um folgende Bereiche gehört: die sog. Realienlexeme (so meint z. B. der englische 
Begriff government das Kabinett, der USTerminus dagegen den gesamten Staatsapparat der drei Gewalten), kulturspezifische Realia und Sachverhalte (historisch-geographische Bezeichnungen und Namen (z. B. Abendland, Morgenland), politische Termini (Rote Armee/Sowjetarmee usw.), Symbolbedeutungen und symbolische Ausdrucksformen (Farbsymbolik z. B. schwarz oder weiß als Trauerkleidung, was dadurch nivelliert werden kann, dass man den Ausdruck: Sie trug schwarz in andere Sprachen mit ,Sie trug Trauerkleidung' übersetzt werden kann; Blumen- und Tiersymbolik usw. 2) Das kommunikativ-prozedurale Wissen umfasst das Interaktionswissen, nämlich das Wissen um soziales Verhalten in bestimmten Situationen (Spechakte wie Bitten, Entschuldigen usw.) und Kommunikationsformen sowie Verbalisierungsgrad, d. h. das Verhältnis zwischen Verbalisiertem und Implizitem (Der Translator geht nicht von der Frage aus „Wie heißt das auf Französisch oder Japanisch“? sondern: „Mit welchen sprachlichen oder außersprachlichen Mitteln realisiert der ZT-Empfänger diese Intention in gegebener Situation?“. 3) Zum textuellen Wissen gehören Kenntnisse von Textsorten und Gebrauchsnormen (kommunikativ-angemessene Formeln, Phraseologismen usw.). 4) Die prognostischen Annahmen des Translators und die kognitiven Strategien umfassen die Verstehensvoraussetzungen des Translators und die Erwartungen der zielsprachlichen Leser sowie die Projektion und die Konstruktion des Fremden (Eigenperspektive und Projektionen, Selbst- und Fremdbilder, kulturelle Stereotype). Diese vier Komponenten stellen ein Matrix der kulturellen Kompetenz dar. Welche dieser Komponenten im Vordergrung stehen, hängt „,von den Arbeitskulturen, von translatorischen Zielsetzungen und von dem cognitive environment des ZT-Lesers, von dem individuell als Situation und Kontext Wahrgenommenen" ab.

Folgende Faktoren müssen dabei beachtet werden: 1) reale Situationskontexte (kulturspezifische Realia, Wissensbestände, Präsuppositionen), 2) Sprachlicher Rede- bzw. Diskurskontext (textkommunikative und textsoziale Konventionen) und 3) Subjektive Wissens- kontexte der kommunizierenden Personen (Anspielungen, Wissens- und Wahrnehmungsmuster, gesellschaftliche Rituale). Die kulturelle Kompetenz spielt eine entscheidende Rolle bei der Erstellung eines gelungenen Translats. Es handelt sich nicht nur darum, dass der/die Translator(in) die "offensichtlichen" kulturspezifischen Elemente in die Zielsprache zutreffend übertragen muss, sondern auch die "verdeckten" kulturspezifischen Ausdrücke, die erst nach dem Aktivieren seines / ihres Hintergrundwissens im kulturellen Kontext als solche erkannt werden (s. Pkt. 4.).

\section{Translatorische Verfahren}

Im Fokus der translatorischen Verfahren steht entweder der Text oder der Prozess oder der/die Translator(in), wobei diese drei Problemkreise in der Fachwissenschaft meistens nicht zusammeneführt werden $/ \mathbf{2 1} /$. Im Folgenden werden ohne Anspruch auf Vollständigkeit translatorische Verfahren dargestellt, die sich auf den Text beziehen: Diejenigen von Kautz /22/ betreffen den Translationsprozess schlechthin, während andere Verfahren sich auf kulturspezifische Elemente beziehen. Nach der Strukturebene werden mikrostrukturelle und makrostrukturelle Ansätze unterschieden $/ 23 / . \mathrm{Zu}$ jenen, welche die Kulturspezifika auf der Wortebene betrachten, gehören die Verfahren z. B. von Reiß /24/, Reiß/Vermeer /25/, Koller /26/ und Newmark /27/, zu diesen, welche sie auf der Textebene behandeln, sind $u$. a. diejenigen von Levý /28/ und Floros/29/ zu rechnen. Kautz/30/ unterscheidet zwischen den Sinngehalt betreffenden und den Stil betreffenden translatorischen Verfahren, die hier mit deutschen, englischen und mazedonischen Beispielen illustriert werden. 1) Translatorische Verfahren, die sich auf den Sinngehalt beziehen, sind: Synonyme: dt. Schaffell > maz. руно / rипо neben овча волна / оvс̌а volna [wörtl.: ,Schaffell']; Antonyme: dt. ich musste lachen > maz. не можев да не се смеам / пе тоžеv da ne se smeam [wörtl.: ,ich konnte nicht nicht lachen']; Expansion (Explikation): dt. Im Westen nichts Neues > engl. All Quiet on the Western Front; Generalisierung: maz. чичко / čičko, вуjко / vujko, тетин / tetin > dt. Onkel; Sonntagszeitung > maz. 
неделен весник/nedelen vesnik [,Sonntagszeitung';,Wochenzeitung']; Konkretisierung: maz. oди дома / odi doma [,nach Hause zu Fuß gehen' oder ,fahren' oder, fliegen'] > dt. nach Hause fahren; Paraphrasierung: dt. verschollen > maz. sy се губи траzama / mu se gubi tragata [,spurlos verschwinden', wörtl.: ,ihm geht die Spur verloren']; usw. 2) Zu den translatorischen Verfahren, die den Stil widerspiegeln, gehören: Metaphorisierung: dt. über etwas hinwegsehen > maz. некому му прогледа низ прсти/nекоти ти progleda niz prsti [,ein Auge zudrücken', wörtl.: ,jdm. durch die Finger sehen']; Demetaphorisierung: dt. den Nagel auf den Knopf treffen > maz. ja трефне суитината / ja trefne suštinata [wörtl.: ,das Wesentliche treffen']; Kommentierende Paratexte (Glossar, Fußnote): maz. добие nemкa / dobie petka [wörtl.: eine Fünf bekommen'] > dt. eine Eins bekommen (Fußnote: Notenspiegel: Sehr gut 5; Gut 4 usw.); Ersatz von Dialekt durch Jargon oder Standardssprache usw.: ausbaldowern (laut Duden-www: salopp) > извиди / izvidi [standardsprachlich ,aufklären, auskundschaften'].

Nach Reiß/31/müsse der Ausgangstext zunächst einem Texttyp zugeordnet werden, da der Stellenwert der Textelemente innerhalb der Textkonstitution nur aus der Funktion des Ausgangstextes und seinem Status in der Ausgangskultur erschließbar sei und die Funktion des Zieltextes im Kommunikationgeschehen keineswegs mit der des Ausgangstextes übereinstimmen müsse. Sie unterscheidet vier Texttypen: einen informativen (mit dem in erster Linie Inhalt vermittelt werde), einen expressiven (mit dem Inhalte in analoger künstlerischer Organisation vermittelt würden, einen operativen (bei dem Appelfunktion und Persuativstrategien im Vordergrund stünden, die auf Mentalität und Kultur des Zieltextlesers abgestimmt werden müssten) sowie einen multi-medialen Texttyp (dessen Textgestaltung auf eine Interdependenz verschiedener Medien zurückzuführen sei). Reiß /32/ schlägt vier Lösungen für kulturbedingte Übersetzungsprobleme vor: 1 ) Entlehnung nämlich „die Übernahme nicht nur der begrifflichen Vorstellung, sondern auch der ausgangssprachlichen Bezeichnung für eine sozial-ökonomische oder kulturelle Einrichtung oder Erscheinung aus dem ausgangssprachlichen Bereich [...]; 2) die Lehnübersetzung, d. h. die Bildung neuer lexikalischen Einheiten in der Zielsprache; 3) Übernahme des fremdsprachlichen Ausdrucks unter Hinzufügung einer Fußnote; 4) eine erklärende Übersetzung. Koller /33/ betrachtet die kulturspezifischen Elemente im Zusammengang mit der Äquivalenzproblematik. Er unterscheidet mehrere Äquivalenzen, zu denen auch die denotative gehört, die er in Entsprechungstypen unterteilt. Der Äquivalenztyp Eins-zu-Null-Ensprechung stellt „echte Lücken“ im Lexikon der Zielsprache dar. Zur Schließung der Lücken schlägt Koller /34/ folgende Verfahren vor: 1) „Übernahme des ausgangssprachlichen Ausdrucks in die Zielsprache (ggf. in Anführungszeichen): (a) unverändert als Zitatwort (Fremdwort): engl. joint venture $>\mathrm{dt}$. Joint-venture; (b) vollständige oder teilweise Anpassung an die phonetischen, graphemischen und/oder morphologischen Normen der Zielsprache (Lehnwort): schwed. obmudsman > dt. der Ombundsmann [...]“; 2) Lehnübersetzung: "der ausgangssprachliche Ausdruck wird wörtlich (Glied für Glied) in die Zielsprache übersetzt: engl. bomb carpet > dt. Bombenteppich“; 3) "Als Entsprechung zum ausgangssprachlichen Ausdruck wird in der Zielsprache ein bereits in ähnlicher Bedeutung verwendeter Ausdruck gebraucht (Wahl der am nächsten liegenden Entsprechung): engl. performance (Lingustik) $>$ dt. Sprachenverwenung [...]“;4) Der ausgangssprachliche Ausdruck wird in der Zielsprache umschrieben, kommentiert oder definiert (Explikation oder definitorische Umschreibung) [...] engl. non-foods dt. Produkte, die keine Lebensmittel sind; 5) Adaptation: „Unter diesem Verfahren versteht die Stylistique comparée [...] die Ersetzung des mit einem AS-Ausdruck erfaßten Sachverhalts durch einen Sachverhalt, der im kommunikativen Zusammenhang der ZS eine vergleichbare Funktion bzw. einen vergleichbaren Stellenwert hat; aus dem engl. burberry wird ein dt. Lodenmantel $[\ldots]^{\prime \prime}$.

Koller /35/ hebt das Problem der sog. unübersetzbaren Wörter hevor, an denen die sog. prinzipielle Unübersetzbarkeit demonstriert würde (dt. Gemüt, gemütlich > fr. charme, esprit, engl. gentleman), für die es in anderen 
Sprachen nur Teilentsprechungen gäbe. „Immerhin ist in Betracht zu ziehen, daß auch diese unübersetzbarsten der kulturgebundenen Wörter kaum isoliert, sondern meistens in textzusammenhängen vorkommen: Kommunikation geschieht im Allgemeinen in Texten, nicht in einzelnen Wörtern. [...] Der Leser/Hörer konstruiert aus dem sich positiv entwickelnden Sinnganzenn des Textes und in ständiger Rückkoppelung zu seinen eigenen Wissensvoraussetzungen die Bedeutung einzelner Wörter, Sätze und Textabschnitte. Das zunächst ungenau oder vage Verstandene wird im Verlauf der Textlektüre sukzessiver adäquater verstanden"

Obwohl Köller die Relevanz des Textzusammenhangs für das steigende Verständnis der kulturgebundenen Wörter untersteicht, wird diese Idee „bei der methodischen Behandlung von kulturspezifischen Wörtern nicht umgesetzt. [...] Die textuelle Dimension von Wör- tern, insbesondere von kulturspezifischen Elementen, wird zwar prinzipiell erkannt, jedoch gehen die methodischen Verfahren eher von vereinzelten Elementen im Text aus, und die textuelle Dimension kommt bei der methodischen Behandlung zu kurz in der Übersetzung zu kurz"/36/.

Newmark /37/ schlägt folgende translatorische Verfahren für kulturspezifische Elemente (cultural terms) vor: 1) Transference, 2) Cultural equivalent, 3) Neutralisation (i.e. functional or descriptive equivalent), 4) Literal translation, 5) Label, 6) Naturalisation, 7) Componential analysis, 8) Deletion (of redundant stretches of language in non-authoritative texts, especially metaphors and imensifiers), 9) Couplet, 10) Accepted standard translation, 11) Paraphrase, gloss, notes, etc. , 12) Classifier.

Zwischen Reiß, Koller und Newmark, die hier in der Reihenfolge zunehmender Differenziertheit dargestellt sind, bestehen weitegehende Übereinstimmungen /38/.

\begin{tabular}{|c|c|c|}
\hline Reiß & Koller & Newman \\
\hline \multirow[t]{2}{*}{ Entlehnung } & \multirow{2}{*}{$\begin{array}{l}\text { Übernahme des AS-Ausdrucks als } \\
\text { Zitatwort oder als angepasstes } \\
\text { Wort }\end{array}$} & Transference \\
\hline & & Neutralisation \\
\hline \multirow[t]{2}{*}{ Lehnübersetzung } & Lehnübersetzung & \\
\hline & $\begin{array}{l}\text { Ausdruck mit ähnlicher Bedeu- } \\
\text { tung }\end{array}$ & Label \\
\hline \multirow{2}{*}{$\begin{array}{l}\text { Übernahme des fremdsprachlichen } \\
\text { Ausdrucks mit Fußnote }\end{array}$} & \multirow{4}{*}{$\begin{array}{l}\text { Umschriebung, Kommentar oder } \\
\text { Definition }\end{array}$} & Componential analysis \\
\hline & & Couplet \\
\hline \multirow[t]{5}{*}{ erklärende Übersetzung } & & Paraphrase, gloss, notes, etc. \\
\hline & & Classifier \\
\hline & Adaptation & \\
\hline & & Accepted standard translation \\
\hline & & Deletion \\
\hline
\end{tabular}

Markstein /39/ unterscheidet folgende translatorische Lösungen: 1) Der Ausdruck wird unverändert als Zitat-Wort in die Zielsprache übernommen: Westminster Abbey, Pizza; 2) Lehnübersetzung [Lehngut]: Erschaffung eines neuen Wortes nach der semantischen Entsprechung: skyscraper ,Himmelskratzer' Wolkenkratzer; das Dritte Reich > the Third Reich; 3) Analogiebildung: Verwendung eines sinngemäß entsprechenden Wortes: Außenminister > secretary of state; 4) Kommentierende Übersetzung: Kommentierung der im ausgangssprachlichen Wort enthaltenen Bedeutungen: Fußnote, integrierter Kommentar. Ihre Lösungen entsprechen beinahe $1: 1$ den Verfahren von Reiß (s. o.). 
Bei den einzelproblemorientierten Ansätzen stellt Floros folgende Defizite fest. Erstens: obwohl die Bedeutung der Aktvierung des Hintergrundwissens nicht unterschätzt werde, fehle eine methodische Umsetzung der theoretischen Überlegungen. So stünden verschiedene Übersetzungsverfahren zur Auswahl, aber es werde nicht erklärt, wie die kulturellen Elemente überhaupt zu identifizieren seien. Als Beispiel diene das Lexem dt. Beipackzettel, das zunächst nicht als Kulturspezifikum angesehen werde, da es im Englischen den Ausdruck package insert gebe. Ein Problem entstehe, wenn die englische Übersetzung für amrikanische Leser bestimmt sei, da es in Amerika weder Arzneimittelverpackungen noch Beipackzettel gebe, sondern nur Packungsaufschriften. So erweise sich der deutsche Ausdruck erst nach der Aktivierung des Hintergrundwissens als Kulturspezifikum. Zweitens: es sei fast ausschließlich die Rede von Realia, d. h. von Elementen auf der lexikalischen Ebene, was zur Vernachlässigung anderer Ebenen (z. B. der stlistischen) führe. Die Realia seien als vereinzelte Elemente im Text betrachtet, ohne dass dabei der textuelle $\mathrm{Zu}$ sammenhang thematisiert werde.

Die ganzheitlichen Ansätze werden am Beispiel von Levý und Floros dargestellt. Levý /40/ setzt sich mit der literarischen Übersetzung auseinander. Sie hänge vom Sprachmaterial, vom historischen und vom nationalen Milieu ab. Grundsätzlich schlägt er folgende Verfahren vor: Substitution (Anwendung einer Analogie in der eigenen Sprache, wenn gleichzeitig die allgemeinere Bedeutung zum Ausdruck kommt) und Transkripiton (Umschrift, wo die Bedeutung, der allgemeine Faktor ganz ausfällt). Des Weiteren kämen auch eine noch „,heimische, merkmallose, neutrale Analogie", wenn in der Zielsprache kein Äquivalent vorhanden ist und die Ausgangssprache die Illusion des Originalmilieus nicht hervorzurufen vermag, sowie Erläuterung und Andeutung (Fußnoten und innere Erläterungen), wenn manche Bezüge auf das Originalmilieu in einer anderen Gesellschaft nicht verständlich sind" /41/.

Diese Verfahren erinnern an die bereits erwähnten. Levy weicht jedoch insofern von denen $a b$, als er das Funktionieren der Einzelelemente im Ganzen, nämlich das Zusammenspiel zwischen dem Datail und dem Ganzen hervorhebt. Dies ist besonders für die Realia bedeutsam, die „Bestandteile breiterer Kontexte im Lebensmilieu der einzelnen Völker" sind. „Wenn der Wert des Ganzen nicht gleich der Summe seiner Teile ist, sondern einer neuen semantischen Qualität, dann verlangt es das Äquivalent eines ähnlichen Ganzen in der Sprache der Übersetzers" /42/.

Trotz Levýs sowohl mikro- als auch makrostruktureller Erfassung der Kulturspezifik sieht Floros /43/ in dem Umstand einen Nachteil, dass er keine Methodik (Schrittfolge) für die Umsetzung dieses Ansatzes in die Praxis beschreibt. Ähnliche Probleme seien grundsätzlich bei den hermeneutischen Ansätzen festzustellen, die sich fast ausschließlich auf die menschliche Intuition stützen und keine systematische Vorgehensweise beim Übersetzen ausarbeiten.

Diesem Mangel versucht Floros zu behelfen. Von Mudersbachs Konzept des Holons ausgehend, betrachtet er den Text als Kulturprodukt, wobei die Einheiten des Textes als Kulturträger fungieren können, die sich erst nach der Aktivierung des Hintergrundwissens als kulturspezifisch erweisen (2002: 75; s. o. das Beispiel mit dem Beipackzettel). Im Fokus steht der Begriff kulturelle Konstellation im Text, die aus sämtlichen Konkretisierungen eines Kultursystems im Text besteht.

Der Übersetzungsprozess besteht nach Floros /44/ aus folgenden Phasen. 1) Rezeptionsphase: Schritt 1: Erstlektüre des Textes unter holistischen Gesichtspunkten; Schritt 2: Ausflistung und Benennung von Kultursystemen (Auflistung, Zweck, Strukturierung), Schritt 3: Holistische Zweitlektüre des Textes, Schritt 4: Indentifizierubg und Gewichtung kultureller Konstellationen; 2) Transferphase (Vorbereutung der kulturellen Konstellation auf ihre Übertragung in die Zielsprache). Schritt 5: Erstellen der zielsprachlichen Kultursysteme (gleiches Vorgehen wie im Schritt 2); Schritt 6: Vergleich mittels kontrastiver Betrachtung; Schritt 7: Kompatibilitätsprüfung; 3) Reproduktionsphrase Schritt 8: Grundsatzentscheidungen; Schritt 9: Neuvertextung. 
Das Verfahren von Floros soll die oben erwähnte Mängel beheben und eine vollständige und systematische Übertragung der kulturspezifischen Elemente gewährleisten. Jedoch fehlt eine Erklärung, wie relevante kulturspezifische Elemente erkannt und übertragen werden. Unberücksichtigt bleiben Probleme, die durch Defekte ses Ausgangstextes, den Auftrag und die situativen Bedingungen sowie die Kompetenz des Translators verursacht werden /45/.

Schleiermacher /46/ geht von der Fremdheit der Originals aus. Der Übersetzer muss die Distanz zwischen dem Original und dem Leser der Übersetzung überwinden oder wenigstens verringern, um so dem Leser das Verständnis des Originals $\mathrm{zu}$ ermöglichen. Diesbezüglich nennt er zwei Ansätze im Hinblick auf die kutlurspezifischen Elemente: „Entweder der Uebersezer läßt den Schriftsteller möglichst in Ruhe, und bewegt den Leser ihm entgegen; oder er läßt den Leser möglichst in Ruhe und bewegt den Schriftsteller ihm entgegen" /47/. Bei jenem Verfahren, die heutzutage als Verfremdung bekannt ist, werden die kulturspezifischen Elemente aus dem Ausgangstext in die Übersetzung übertragen, während dieses, heutezutage Einbürgerung genannt, ein Ersatz der kulturspezifischen Elemente aus dem Original durch Elemente der zielsprachlichen Kultur darstellt. Sämtliche oben genannte translatorische Verfahren lassen sich mit unterschiedlichen Intensitätsgraden grundsätzlich auf diese beiden Ansätze zurückführen (Verfremdung: Zitatwort, Lehnübersetzung; Explikation oder definitorische Umschreibung uw.; Einbürgerung: Analogiebildung/Adaptation usw.).

Die Übersicht über die translatorischen Verfahren wird hier mit der Feststellung beendet, dass die Rolle des Kontextes nicht unterschätzt werden darf, da er letzten Endes über den Status eines Ausdrucks als kulturspezifischen Elements entscheidet, und dass bei der Wahl zwischen Verfremdung und Einbürgerung jenem Verfahren Vorrang gewährt wird, da die Eigenart der Ausgangskultur auch in der Übersetzung zum Vorschein kommen muss. Die Verwendung der Zielsprache berechtigt nämlich den / die Translator(in) nicht dazu, Elemente der Ausgangskultur einfach durch solche der Zielkultur zu ersetzen, um beim Leser ein mühelose, jedoch kulturell versetzte Lesewese zu ermöglichen.

\section{Korpusanalyse und Methode}

Im Folgenden werden fünf ausgewählte kulturspezifische Elemente in der deutschen Übersetzung von Blaze Koneskis Erzählungen "Һубов/Ljubov", "Liebe“ und „Песна / Pesna“, „Petres Lied“ vor dem Hintegrund der jeweiligen Kultursubsysteme erörtert, zu denen die jeweiligen Ausdrücke gehören.

\subsection{Maz. Песна / Pesna $>$ dt. Petres Lied}

Es handelt sich um den Titel der Erzählung Песна / Pesna > Petres Lied. Titel erfüllen 1) eine distinktive Funktion (sie sind Namen, die durch Benennung eine Größe gegen andere abgrenzen) sowie sämtliche Sprachfunktionen nach dem Kommunikationsmodell Jakobsons /48/, und zwar: 2) eine metatextuelle Funktion, derzufolge die Art des (Kunst-)Werks angegeben wird, und zwar implizit (bei einem hohen Bekanntheitsgrad) oder explizit (durch Angabe der Art des (Kunst-)Werks: Faust. Eine Tragödie; 3) eine phatische Funktion, die den Kommunikationskanal betrifft (z. B. in Bibliothekskatalogen); 4) eine referenzielle (denotative, Darstellungs-) Funktion, die dem Rezipienten Auskunft über Inhalteslemente verleit, die jedoch nicht immer vorliegt (aus dem Titel lässt sich nicht immer auf den Inhalt schließen); 5) eine poetische Funktion, die den sprachlichen Ausdruck betrifft; 6) eine Appelfunktion (konative Funktion), die darauf abzielt, den potentiellen Rezipienten dazu zu bewegen, das (Kunst)Werk zu rezipieren; 7) eine expressive (emotive) Funktion, die Information über den auktorialen Erzähler vermittelt (serb.-kroat. Немам више времена/Nemam više vremena, wörtl. 'Ich habe keine Zeit mehr' /49/, /50/.

Durch die Übernahme der slawischen Variante Пempe/Petre (lat. Petrus, dt. u. a. Peter, Petrus) wird der Leser bereits vor dem Lesen 
des Textes an die fremde Kultur herangeführt. Dies erfüllt die Appelfunktion.

Beim Gattungsnamen песна / pesna handelt es sich um Kollers denotative Divergenz (seinen Äquivalenztyp Eins-zu-viele-Entsprechung), da das angeführte Lexem mindestens diese Bedeutungen hat: ,Lied' und ,Gedicht'. Der mazedonische Titel ist im Hinblick auf die Art des Kunstwerkes irreführend, da Blaze Koneski einige seiner Gedichte so betitelt hat (wo die metatextuelle Funktion in der Bedeutung ,Gedicht' überwiegt). Beim Titel der Erzählung Песна / Pesna handelt es sich also vor allem um die referenzielle Funktion und nicht um die metatextuelle Funktion des Titels.

Was das Übersetzungsverfahren von Titeln betrifft, liegt hier formale Expansion und semantische Reduktion vor: die Einfügung des sächsischen Genitivs (Petres) stellt eine lexikalische Erweiterung dar, welche durch diese zusätzliche Auskunft den semantischen Bereich des Nomens einschränkt. Eine weitere Einschränkung ergibt sich aus der denotativen Divergenz, nämich der Monosemierung des Lexems песна / pesna (s. о.).

\subsection{Maz. paкuja/rakija $>$ dt. Rakija}

Dieses kulturspezifische Element kommt im folgenden Auszug vor:

Пред него на прозорчето стои шише ракија ${ }^{1}$, го донесе да се опие, но одвај е отпразнето (Песна, 41). / Pred nego na prozorčeto stoi šiše rakija, go donese da se opie, edvaj e otprazneto.

Vor ihm am Fenster steht ein Gläschen Rakija, er hat es mitgebracht, um sich zu betrinken, hat aber bloß daran genippt (Petres Lied, 302).

Es handelt sich um das Subsystem der Trinkkultur. Laut Duden (-www) hat das türkische Lehnwort Raki folgende Bedeutung: ,türkischer Branntwein aus Anis und Rosinen'. Es entstammt dem Arabischen (türkisch rakl $<$ arabisch `araq, im Deutschen entlehnt als Arrak mit

\footnotetext{
${ }^{1}$ Hevorhebung der Verf. hier und in folgenden Zitaten aus Koneskis Erzählungen.
}

der Bedeutung: ,[ostindischer] Branntwein aus Reis oder Melasse'.

Das türkische Lehnwort paкuja / rakija hat im Mazedonischen die Bedeutung ,Schnaps'. Das alkoholische Getränk Rakija wird in Nordmazedonien in der Regel aus Weintrauben hergestellt. Wenn Anis hinzugegeben wird, werden andere Ausdrücke verwendet (z. B. мастика / mastika, анасонка / anasonka).

Die Rakija hat einen besonderen Stellenwert in den Ess- und Trinkgewohnheiten auf dem Balkan und wird als Nationalgetränk Nordmazedoniens angesehen. Sie wird als Aperitif (und nicht als Digestif) zu Mezze getrunken, bevor beim Hauptgericht oft zu Wein oder Bier übergegangen wird. Oft verbindet man damit ein geselliges Beisammensein, was jedoch im Kontext der Erzählung nicht der Fall ist. Die Übersetzerin hat sich also für die Übertragung dieses die Ess- und Trinkkultur auf dem Balkan prägendes Element aus dem Original und die Übersetzung und somit auf dessen Relevanz hingewiesen.

Zur leserfreundlichen Verdeutlichung des verfremdenden Ausdrücks Rakija wird hier anstatt eines Glossareintrags, die in der Übersetzung zu finden ist, eine in den Text eingebettete Erklärung vorgeschlagen.

Vor ihm am Fenster steht eine Flasche Weintraubenbrand Rakija, das mazedonische Nationalgetränk, er hat sie mitgebracht, um sich zu betrinken, hat aber bloß daran genippt.

\subsection{Maz. Богоројu̧a/Bogorojca $>$ dt. Mariä Hilmmelfahrt}

Folgendes Zitat enthält das oben erwähnte Geortonym:

Соколе решува да побрза со грозјебрањето, веќе сме кај Богоројца, [...] (Һубов/ Ljubov, 274) / Sokole resuva da pobrza so grozjebranjeto, veke sme kaj Bogorojca, [...].

Sokole beschloss, die Weinlese möglichst bald zu beginnen, es war schon 
gegen Mariä__Hilmmelfahrt; [...] (Liebe, 535).

Dieses Element des Kultursubsystems der kirchlichen Feiertage erfordert eine Aktivierung des Hintergrundwissens des Übersetzers / der Übersetzerin, und zwar unter mindestens drei Aspekten. Erstens ist hier der ausgangssprachliche Ausdruck mehrdeutig und er muss präzisiert werden, da es ich um Kollers denotative mazedonisch-deutsche Divergenz handelt (s. Pkt. 5.1.). Die dialektale Variante des Feiertagsnamens Богоројųа/Bogorojca (standardsprachlich: Богородицุa / Bogorodica), wörtl.: ,Mutter Gottes' bezieht sich sowohl auf Mariä Geburt als auch auf Mariä Himmelfahrt. Zweitens muss dem/der Übersetzer(in) der Zeitraum der Weinlese in Nordmazedonien bekannt sein. Sie beginnt zwar für bestimmte Tafelsorten schon im Ausgust, aber "offiziell" wird sie erst Anfang September eröffnet. Drittens spielt der Umstand eine Rolle, dass in den deutschsprachigen Ländern der gregorianische Kalendar verwendet wird, während in Nordmazedonien die kirchlichen Feiertage nach dem julianischen Kalendar festleget werden, dem zufolge sie 13 Tage später erfolgen (Mariä Himmelfahrt: 15. bzw. 28 Augist; Mariä Geburt: 8. bzw. 21. September). Aus dem Kontext der Erzählung ergibt sich, dass die Weinlese dem Feiertag vorausgehen sollte. In der Erzählung geht es darum, dass die Gestalt Sokole sich mit der Weinlese zu beeilen beabsichtigt, da Mariä Geburt, der 21. September, heranrückt. Da in der Übersetzung der Feiertag Mariä Hilmmelfahrt vorkommt, wird der Leser wahrscheinlich die Zeit um Mitte August annehmen. Diese übersetzerische Entscheidung kann eine Folge auch dessen sein, dass die Übersetzerin anstatt die Notwenigkeit, sich mit der Weinlese zu beeilen, die Notwendigkeit, mit der Weinlese zu beginnen, ausgedrückt hat. Dieser Zeitpunkt, Mitte August, muss jedoch originalgetreu noch zweimal „verschoben" werden: um den Zeitunterschied zwischen Mariä Himmelfahrt und Mariä Geburt (23 Tage) und um den Zeitunterschied zwischen dem gregorianischen und dem julianischen Kalendar (13 Tage). So wurde in der Übersetzung auf Mitte August anstatt auf den
21. September hingewiesen. Obwohl diese Abweichung manchem Leser nicht schwerwiegend erschienen mag, ist die Notwendigkeit der Berücksichtung derartiger kultureller Unterschiede durchaus erwähnenswert. Folgende übersetzerische Lösung wird vorgeschlagen:

Sokole beschloss, sich mit der Weinlese zu beeilen, es war schon gegen Mariä Geburt, den 21. September.

\subsection{Maz. opo / oro $>$ dt. Kolo}

Folgende Beispiele belegen das Lexem opo / oro.

Орото се растура (Песна / Pesna, 46) / Oroto se rastura.

Der Kolo löst sich auf (Petres Lied, 307 ).

Иди, самовилска ќерко, иди, играј самовилско оро (Песна / Pesna, 44) / Idi, samovilska kerko, idi, igraj samovilsko oro.

Geh, Vilatochter, geh und tanze den Vilareigen, und dein leichter Fuß wird nicht auf die Wiese treten (Petres Lied, 305).

Es handelt sich um das Subsystem der Tanzkultur. Das Lexem opo / oro kommt in der Erzählung neunmal vor. Siebenmal hat es die Grundbedeutung (Volkstanz), und zweimal wird es metaphorisch verwendet (самовилско opo / samovilsko oro, dt. ,Feentanz'). Im ersten Fall wird es mit Kolo wiedergegeben, im zweiten Fall mit Vilareigen.

Laut Duden (-www) hat Kolo (slawisch für ,Rad') die Bedeutungen: ,auf dem Balkan verbreiteter Kettenreigentanz in schnellem 2/4Takt' und ,Nationaltanz der Serben'. Dieses Lehrwort entstammt nicht der Ausgangssprache, sondern einer ihr nahe liegender Sprache: dem damaligen Serbokroatischen. Die Übersetzerin muss wohl davon ausgegangen sein, dass die ausgangssprachliche Kultur Berührungspunkte vor allem mit der serbischen, aber auch mit der kroatischen (sowie mit anderen Kulturen der Balkanländer z. B. mit der bulgarischen) aufweist, und das Lexem in der zweiten Bedeutung verwendet haben, auf die im Glossar verweisen wird. Das Lexem коло / kolo gibt es auch 
im Mazedonischen, jedoch standardsprachlich nicht im Zusammenhang mit demTanz, sondern mit den Bedeutungen ,Rad', ,Runde' usw. /51/. Umgangssprachlich wird es jedoch auch mit dieser Bedeutung verwendet. Es handelt sich hier um eine Entlehnung aus einer Drittsprache, die der Ausgangssprache nahe liegt.

Bei der Analyse der Metapher самовилско оро / samovilsko oro steht das Lexem opo/oro im Vordergrund. Deren Übersetzung mit Vilareigen stellt eine Generalisierung dar, da es sich hier um einen Feentanz handelt, der offensichtlich als nicht so stark an einen bestimmten Ort gebunden gesehen wird. Dennoch geht die Vorstellung eines Rundtanzes nicht verloren.

Es scheint nicht gerechtfertigt, ein Element der Ausgangskultur durch eines einer benachbarten Kultur zu ersetzen. Andererseits würde die Übernahme von maz. opo / oro in die Übersetzung wohl zu künstlich klingen. Außerdem könnte der Leser durch das Homonym griech. Oro-, oro- (,Berg, Gebirge') verleitet werden. Hier wird als übersetzerische Lösung Reigen vorgeschlagen, da sie trotz der Generalisierung das Wesen des Tanzes wiedergibt. Der zielsprachliche Ausdruck hat also Vorrang vor dem Ausdruck aus einer benachbarten Sprache.

Der Reigen löst sich auf.

\subsection{Maz. чардак / с̌ардак > dt. Maisscheune}

Folgendes Zitat enthält dieses kulturspezifische Element:

Во манастирскиот двор под оревите, долу покрај реката, по чардаците, сегде каде што има сенка преполно е исполегнат народ (Песна / Pesna, 41).

Im Klosterhof unter den Nußbäumen, unten am Fluß, bei den Maisscheunen, überall, wo Schatten ist, hat sich viel Volk gelagert (Petres Lied, 302).

Das kulturelle Subsystem betrifft das Bauwesen. Das aus dem Türkischen entlehnte Wort hat im Mazedonischen die Bedeutung:
,Diele, Vorraum im oberen Stockwerk in Häusern älteren Typs' /52/. Im Wörterbuch der Donauschwäbischen Wirtschaft ist Tschardak als Synonym zu Hambar angeführt mit der Bedeutung: ,überdachter Bretter- und Rutenbau, manchmal mit Schlafmöglichkeit davor, zum Aufbewahren von Weizen oder Bohnen'. „Das Substantiv gelangte von türk. çardak ,Laubengang, Pergola' über bulg., serbokr. und albanisch ciardak, ceardak ,Veranda' in die donauschwäbischen Dialekte“ /53/. Diese Bedeutung ist auch bei Grčeva und Rau /54/ angeführt: ,Veranda, (großer) Balkon'.

Das vorliegende Beispiel zeigt, dass das Zurückgreifen auf die Bedeutung dieses Lexems in anderen Balkanregionen (hier im Gebiet der Donauschwaben) zu abweichenden Ergebnissen führt. In Blaze Koneskis Erzählung wird eine Szene beschrieben, wo die Menschen sich auf die Holzveranden gelegt haben.

Am schwierigsten ist es wohl, das Rustikale hinüberzubringen, insbesondere vor dem Hintergrund des vorgeschlagenen Lexems $\mathrm{Ve}^{-}$ randa. In einem gewissen Grad kann das vielleicht durch die Erwähnung des Baumaterials erreicht werden. Obwohl im Duden (-www) das Stichwort Tschardak nicht vorkommt, käme im Sinne der Verfremdung eine Übersetzung mit hinzugefügter Erklärung in Frage, z. B.:

Im Klosterhof unter den Nußbäumen, unten am Fluß, auf den sog. Tschardaks, den Holzveranden, überall, wo Schatten ist, hat sich viel Volk gelagert

\section{Schlussfolgerung}

In Pkt. 5 wurden folgende kulturspezifische Elemente behandelt: maz. Песна / Реsna > dt. Petres Lied, maz. paкuja / rakija > dt. Rakija, maz. Богоројu̧a/Bogorojca > dt. Mariä Hilmmelfahrt, maz. opo / oro > dt. Kolo, maz. чардак / с̌ардак > dt. Maisscheune, die sich nach dem semantichen Kriterium einteilen lassen. Die Übersetzungen ins Deutsche unterliegen einer Prüfung des formalen und des Referenzumfangs. 


\begin{tabular}{|c|c|c|c|c|}
\hline $\begin{array}{c}\text { Original > Überset- } \\
\text { zung } \\
(1967,1972)\end{array}$ & Semantik & $\begin{array}{l}\text { Formaler Umfang } \\
\text { der Übersetz. }\end{array}$ & $\begin{array}{l}\text { Referenzüber- } \\
\text { einstimmung } \\
\text { der Übersetz. }\end{array}$ & $\begin{array}{l}\text { Referenz- } \\
\text { umfang der } \\
\text { Übersetz. }\end{array}$ \\
\hline $\begin{array}{l}\text { Песна / Pesna> } \\
\text { Petres Lied }\end{array}$ & Titel & Expansion & vorhanden & Reduktion \\
\hline paкuja / rakija > Rakija & Getränkename & gleichbleibend & vorhanden & gleichbleibend \\
\hline $\begin{array}{l}\text { Богоројu̧а / } \\
\text { Bogorojca > Mariä } \\
\text { Hilmmelfahrt }\end{array}$ & Feiertagsname & Expansion & nicht vorhanden & Reduktion \\
\hline opo / oro $>$ Kolo 1 & Tanzname & gleichbleibend & bedingt vorhanden & $\begin{array}{l}\text { bedingte Ex- } \\
\text { pansion }\end{array}$ \\
\hline opo / oro >-reigen 2 & & & vorhanden & Expansion \\
\hline $\begin{array}{l}\text { чардак / с̌ардак> } \\
\text { Maisscheune }\end{array}$ & Hausteilname & gleichbleibend & nicht vorhanden & abweichend \\
\hline
\end{tabular}

Die formale Dimension liegt im Spannungsfeld zwischen gleichbleibendem und expandierendem Umfang. Im Hinblick auf die Referenz liegen drei Abweichungen vor. Beim Feiertagsnamen, der im Original zwei Feiertage umfasst und der augrund des Kontextes in der Übersetzung spezifiziert werden muss, wurde eine nicht zutreffende übersetzerische Lösung gewählt. Eine bedingt vorhandene Referenzübereinstimmung ist beim Tanznamen 1 und eine fehlende Referenzübereinstimmung ist beim Hausteilnamen festzustellen, die jedoch anders bedingt sind: bei jenem wurde ein Lehnwort aus einer Drittsprache herangezogen, bei diesem wurde das aus dem Türkischen in die Ausgangssprache entlehnte Lexem verwendet, jedoch mit einer von der Ausgangssprache abweichenden Bedeutung.

Der Referenzumfang der Übersetzung ist reduziert, gleichbleibend oder expandiert. Die Reduktion erfolgt beim Titel durch die Hinzufügung des Personennamens und beim Feiertagsnamen durch die Spezifizierung. Der Getränkenam weist keine Abweichungen auf. Beim Hausteilnamen wurde ein Referent ausgedrückt, der dem Original nicht entspricht. Die Übersetzung des Tanznamens 1, 2 inkludiert (bedingt) die Tanzart opo / oro in den Oberbegriff.

Die weitere Betrachtung der Übersetzungen umfasst die kulturspezifische Dimension.

\begin{tabular}{|c|c|c|c|}
\hline $\begin{array}{c}\text { Original }>\text { Übersetzung } \\
(1967,1972)\end{array}$ & $\begin{array}{l}\text { Kulturspezifik im } \\
\text { Original }\end{array}$ & $\begin{array}{l}\text { Sichtbarkeit der } \\
\text { Kulturspezifik im } \\
\text { Original }\end{array}$ & $\begin{array}{l}\text { Übersetz. Umgang mit der } \\
\text { Kulturspezifik }\end{array}$ \\
\hline $\begin{array}{l}\text { Песна / Pesna > } \\
\text { Petres Lied }\end{array}$ & nicht vorhanden & - & $\begin{array}{l}\text { ausgangsorientierte Verfrem- } \\
\text { dung (Zitatwort) }\end{array}$ \\
\hline paкuja / rakija > Rakija & vorhanden & sichtbar & $\begin{array}{l}\text { ausgangskulturorientierte Ver- } \\
\text { fremdung (Zitatwort) }\end{array}$ \\
\hline $\begin{array}{l}\text { Богоројųа / } \\
\text { Bogorojca }>\text { Mariä } \\
\text { Hilmmelfahrt }\end{array}$ & vorhanden & nicht sichtbar & Einbürgerung / Neutralisierung \\
\hline opo / oro $>$ Kolo 1 & vorhanden & sichtbar & $\begin{array}{l}\text { drittkulturorientierte Verfrem- } \\
\text { dung (Zitatwort) }\end{array}$ \\
\hline opo / oro >-reigen 2 & & & Einbürgerung / Neutralisierung \\
\hline
\end{tabular}




\begin{tabular}{|l|l|l|l|}
\hline $\begin{array}{l}\text { чардак / čapdaא> } \\
\text { Maisscheune }\end{array}$ & vorhanden & sichtbar & Einbürgerung / Neutralisierung \\
\hline
\end{tabular}

Beim Titel wurde in die Übersetzung ein kulturspezifisches Element eingefügt, und zwar den unveränderten Personennamen aus dem Originaltext, was dem Titel eine verfremdende Dimention verleiht. Das ist ein Schritt weiter in Richtung Verfremdung als die bloße Übernahme eines kulturspezfischen Elements und seine Verwendung an derselben Stelle wie im Ausgangstext. Der Feiertagsname illustriert eine "verdeckte“ Kulturspezifik. Dadurch, dass in den beiden Kulturen christliche Feiertage begangen werden, wurde die Übersetzerin dazu verleitet, sich für einen der zwei in Frage kommenden Begriffe zu entscheiden, ohne dabei die zeitliche Situierung der Weinlese und den in der Ausgangskultur verwendeten Kalendar sowie den Kontext ausreichend zu berücksichtigen. Der überwiegende Teil der verfremdenden übersetzerischen Entscheidungen entspricht der Stellungname Schleiermachers, der hervorhebt, dass bestimmte Gedanken dermaßen eng an eine Sprache gebunden sind, dass eine Übersetzung notgedrungen in einem gewissen Umfang künstlich klingt. Diese Bindung ist so stark, dass nicht möglich ist, auf die Frage zu antworten, wie ein Text aussehen würde, wenn der Autor ihn in einer anderen Sprache verfasst hätte. Ja, was will man einwenden, wenn ein

\begin{tabular}{|c|c|}
\hline $\begin{array}{c}\text { Original > Übersetzung } \\
(1967,1972)\end{array}$ & Zustimmung oder Voschlag durch Verf. \\
\hline Песна / Pesna > Petres Lied & Petres Lied \\
\hline paкuja / rakija > Rakija & Rakija (mit Erklärung) \\
\hline Богоројцุа /Bogorojca $>$ Marï__Hilmmelfahrt & Mariä Geburt (mit Erklärung) \\
\hline opo / oro > Kolo 1 & \multirow[t]{2}{*}{ Reigen/-reigen } \\
\hline opo / oro >-reigen 2 & \\
\hline чардак / с̌ардак > Maisscheune & Holzveranda \\
\hline
\end{tabular}

Uebersezer dem Leser sagt, Hier bringe ich dir das Buch, wie der Mann es geschrieben haben würde, wenn er es deutsch geschrieben haben würde; und der Leser ihm antwortet, Ich bin dir eben so verbunden, als ob du mir des Mannes Bild gebracht hättest, wie er aussehen würde, wenn seine Mutter ihn mit einem anderen Vater erzeugt hätte? Denn wenn von Werken, die in einem höheren Sinne der Wissenschaft und Kunst angehören, der eigenthümliche Geist des Verfassers die Mutter ist: so ist seine vaterländische Sprache der Vater dazu. /55/

Der / die Übersetzer(in) hat zu entscheiden, inwieweit er / sie verfremdende Elemente in die Übersetzung aufnimmt, um ein bestimmtes $\mathrm{Maß}$ an Verträglichkeit beim Lesen nicht zu überschreiten. Eine andere nicht weniger wichtige Dimension ist das Kennenlernen der Ausgangskultur, das eine Übersetzung durch Verfremdung erforderlich macht. Der/ die Übersetzer(in) muss also zwischen sprachlich Natürlichem und Ästhetischen einerseits und Edukativem andererseits balancieren.

Im Zusammenhang mit den hier erörterten Ausdrücken werden folgende übersetzerische Entscheidungen vorgeschlagen:
Die vorliegende Untersuchung bestätigt die Feststellung von Kučiš /56/: „[Ü]ber Jahrhunderte kreisten zahlreiche Diskurse, Abhandlungen und Polemiken um eine translatologische Schlüsselfrage, die durch die Dichotomie, wörtliches oder kontextuelles' bzw. ,produktives oder reproduktives' gekennzeichnet ist." Sie hebt mit Recht hervor /57/ dass „die Frage des wörtlichen, getreuen, des formalen oder freien, des einbürgernden oder kontextuellen Übersetzens [sich] wie ein roter Faden durch die Geschichte des Übersetzens [zieht] und [...] auch im Zentrum aller Versuche [steht], die Translation als Wissenschaftsdisziplin zu begründen, wobei sich alle bisher aufgestellten Überset- 
zungstheorien auf die praktische Herangehensweise an das Problem stützen und in ihr gründen." Es besteht jedoch die Hoffnung, dass mit den vorgeschlagenen übersetzerischen Lösungen ein Gleichgewicht zwischen der Leserlichkeit (da ein mehr oder weniger gekünstelter Ausdruck eine unvermeidliche Folge der Verfremdung ist) und der Vergegenwärtigung der fremden Kultur in der Übersetzung einigermaßen erreicht wurde.

Notes

/1/ Kučiš, Vlasta (2017). Eine Reise durch die Geschichte der Translation. In: Kučiš, Vlasta (Hrg.), Žagar-Šoštarić, Petra (Hrg.). Beiträge zur Translation von gestern, heute und morgen. Rijeka: Filozofski fakultet Sveučilišta. 2017, 12-28 (http://izdavastvo.ffri.hr/wp-content/ uploads/2017/01/END_WEB_VERSION.pdf (23.4.2018).

/2/ Koller, Werner ('2001). Einführung in die Übersetzungswissenschaft. 6. durchges. und aktualisierte Aufl. Wiesbaden: Quele und Meyer (1979).

/3/ Newmark, P. (2001). A Textbook of Translation. Shanghai: Shanghai Foreign Language Education Press (1988).

/4/ Ibid.

/5/ Floros, Gorgos (2003). Kulturelle Konstellationen in Texten: zur Beschreibung und Übersetzung von Kultur in Texten. Tübungen: Narr.

/6/ Kupsch-Losereit, S. (2003). Die kulturelle Kompetenz des Translators. Hauptseite von Frau Dr. Kupsch-Losereit. Johannes Gutenberg-Universität Mainz. Letzte Bearbeitung: 8.1.2003 (http://www.fask.unimainz.de/user/kupsch/kompetenzkulturell.html; 25.3.2018).

/7/ Mudersbach, K. (2002). Kultur braucht Übersetzung. Übersetzung braucht Kultur (Modell und Methode). In: Thome, G. et. al.: Kultur und Übersetzung: methodologische Probleme des Kulturtransfers : mit ausgewählten Beiträgen des Saarbrücker Symposiums 1999. Tübingen: Narr (= Jahrbuch Übersetzen und Dolmetschen, Bd. 2).

/8/ Ibid.

/9/ Ibid.

/10/ Koller, Werner (62001). Einführung in die Übersetzungswissenschaft. 6. durchges. und aktualisierte Aufl. Wiesbaden: Quele und Meyer (1979).

/11/ Albrecht, J. (2005): Übersetzung und Linguistik. Tübingen: Narr.

/12/ Reiß, K. (1971). Möglichkeiten und Grenzen der Übersetzungskritik: Kategorien und Kriterien für eine sachgerechte Beurteilung von Übersetzungen. München: Hueber (= Foreign Language Study)

/13/ Markstein, Elisabeth (1998). „Realia“. In: SnellHornby, Mary / Hönig, Hans G. / Kußmaul, Paul / Schmitt, Peter A. (Hg.): Handbuch Translation (= Stauffenburg Handbücher). Tübingen: Narr, S. 288-291.

/14/ Ibid.

/15/ Kutz, Wladimir (1981). Zur Auflösung der Nulläquivalenz rzússischsprachiger Realienbenennungen im Deutschen. In: Kade, Otto (Hg.): Probleme des übersetzungswissenschaftlichen Textvergleichs (= Übersetzungswissenschfatliche Beiträge 4). Leipzig: Enzyklopädie, S. 106-138.

/16/ Wurm, A. (2013). Eigennamen und Realia in einem Korpus studentischer Übersetzungen (KOPTE). trans-kom6 [2], 381-419.

/17/ Tuovinen, S. (2011). Eigennamen und andere Realienbezeichnungen beim Übersetzen eines deutschen Kinderbuchs ins Finnische. Am Beispiel von Rico, Oskar und Die Tieferschatten von Andreas Steinhöfel (Magisterarbeit). Universität Jyväskylä (https://jyx.jyu.fi/dspace/ bitstream/handle/123456789/36986/URN_NBN_f i_jyu-2011112311719.pdf?sequence $=5$; Zugriff: 28.4.2018).

/18/ Kučiš, Vlasta (2016). Translatologija u teoriji $i$ praksi. Zagreb: HKD.

/19/ Wotjak, Gerd (1993). „Interkulturelles Wissen und zweisprachig vermittelte Kommunikation“, Revista de Filología Alemana 1, 181-196 (file:///C:/Users/user/ Documents/Translation/Wotjak,\%20Gerd,\%20Interkulturelles\%20Wissen,\%20statija.PDFÖ; 25.3.2018).

/20/ Kupsch-Losereit, S. (2003). Die kulturelle Kompetenz des Translators. Hauptseite von Frau Dr. Kupsch-Losereit. Johannes Gutenberg-Universität Mainz. Letzte Bearbeitung: 8.1.2003 (http://www.fask.uni-

mainz.de/user/kupsch/kompetenzkulturell.html; 25.3.2018).

/21/ Müller, Ina (2008). Die Übersetzung von Abstracts aus translationswissenschaftlicher Sicht (RussischDeutsch-Englisch). Eine Untersuchung am Beispiel von Abstracts aus russischen Fachzeitschriften zur Schweisstechnik und ihren Übersetzungen ins Deutsche und Englische. Berlin: Frank und Trimme. (https://books.google.mk/books?id=a3iYPJgmi1g $\mathrm{C} \&$ printsec $=$ frontcover\&source $=g b s \_g e \_s u m-$ mary_r\&cad $=0 \# \mathrm{v}=$ onepage \& $\mathrm{q} \& \mathrm{f}=$ false; 25.3.2018)

/22/ Kautz, U. (2000). Handbuch Didaktik des Übersetzens und Dolmetschens. München: Iudicum. 
/23/ Floros, Gorgos (2003). Kulturelle Konstellationen in Texten: zur Beschreibung und Übersetzung von Kultur in Texten. Tübungen: Narr.

/24/ Reiß, K. (1971). Möglichkeiten und Grenzen der Übersetzungskritik: Kategorien und Kriterien für eine sachgerechte Beurteilung von Übersetzungen. München: Hueber (= Foreign Language Study)

/25/ Reiß, K. und Vermeer, H. J. (1991). Grundlegung einer allgemeinen Translationstheorie. 2. Aufl. (= Linguistische Arbeiten 147). Darmstadt: Niemeyer.

/26/ Koller, Werner ( $\left.{ }^{6} 2001\right)$. Einführung in die Übersetzungswissenschaft. 6. durchges. und aktualisierte Aufl. Wiesbaden: Quele und Meyer (1979).

/27/ Newmark, P. (2001). A Textbook of Translation. Shanghai: Shanghai Foreign Language Education Press (1988).

/28/ Levý, J. (1969). Die literarische Ubersetzung. Theorie einer Kunstgattung. Frankfurt a. M und Bonn: Athenäum.

/29/ Floros, Gorgos (2003). Kulturelle Konstellationen in Texten: zur Beschreibung und Übersetzung von Kultur in Texten. Tübungen: Narr.

/30/ Kautz, U. (2000). Handbuch Didaktik des Übersetzens und Dolmetschens. München: Iudicum.

/31/ Reiß, K. (1971). Möglichkeiten und Grenzen der Übersetzungskritik: Kategorien und Kriterien für eine sachgerechte Beurteilung von Übersetzungen. München: Hueber (= Foreign Language Study)

/32/ Ibid

/33/ Koller, Werner (62001). Einführung in die Übersetzungswissenschaft. 6. durchges. und aktualisierte Aufl. Wiesbaden: Quele und Meyer (1979).

/34/ Ibid.

/35/ Ibid

/36/ Floros, Gorgos (2003). Kulturelle Konstellationen in Texten: zur Beschreibung und Übersetzung von Kultur in Texten. Tübungen: Narr.

/37/ Newmark, P. (2001). A Textbook of Translation. Shanghai: Shanghai Foreign Language Education Press (1988).

/38/ Floros, Gorgos (2003). Kulturelle Konstellationen in Texten: zur Beschreibung und Übersetzung von Kultur in Texten. Tübungen: Narr.

/39/ Markstein, Elisabeth (1998). „Realia“. In: SnellHornby, Mary / Hönig, Hans G. / Kußmaul, Paul / Schmitt, Peter A. (Hg.): Handbuch Translation (= Stauffenburg Handbücher). Tübingen: Narr, S. 288-291.

/40/ Levý, J. (1969). Die literarische Ubersetzung. Theorie einer Kunstgattung. Frankfurt a. M und Bonn: Athenaüm.

/41/ Ibid

/42/ Ibid
/43/ Floros, Gorgos (2003). Kulturelle Konstellationen in Texten: zur Beschreibung und Übersetzung von Kultur in Texten. Tübungen: Narr.

/44/ Ibid

/45/ Müller, Ina (2008). Die Übersetzung von Abstracts aus translationswissenschaftlicher Sicht (RussischDeutsch-Englisch). Eine Untersuchung am Beispiel von Abstracts aus russischen Fachzeitschriften zur Schweisstechnik und ihren Übersetzungen ins Deutsche und Englische. Berlin: Frank und Trimme.

(https://books.google.mk/books?id=a3iYPJgmi $1 \mathrm{gC} \&$ printsec $=$

frontcover\&source=gbs_ge_summary_r\&cad $=0 \# \mathrm{v}=$ onepage $\& \mathrm{q} \& \mathrm{f}=$ false; 25.3.2018)

/46/ Schleiermacher, F. (1963 [1918]). Ueber die verschiedenen Methoden des Uebersezens. In: H. J. Störig (Hg.): Das Problem des Übersetzens. Darmstadt: Wissenschaftliche Buchgesellschaft, 3870

(file://C:/Users/user/Documents/Translation/S chleiermacher.pdf; Zugriff: 28.4.2018).

/47/ Ibid.

/48/ Jakobson, R. (2000). On linguistic Aspects of Translation, Venuti, Lawrence (ed): The Translation Studies Reader, London / New York: Routledge, (1959), 113-118.

/48/ Bojkovska, Е. (2016а) / Бојковска, Е. Превођење наслова књижевних дела“, Културе у преводу. Зборник радова конференције, Београд 4-5 јуна 2015. Књига 2. Београд: Универзитет у Београду. Филолошки факултет, стр. 201215.

[Bojkovska, E. (2016a) / Bojkovska, E. Prevođenje naslova književnih dela“, Kulture u prevodu. Zbornik radova konferencije, Beograd 4-5 juna 2015. Kniga 2. Beograd: Univerzitet $u$ Beogradu. Filološki fakultet, str. 201-215.]

/50/ Bojkovska, E (2016b). Nähe und Distanz zwischen Original und übersetztem Titel im Hinblick auf ihre Identität. In: M. Petrović Jülich und N. Vujčić (Hg.): Nähe und Distanz in Sprache, Literatur und Kultur. Beiträge zur 8. Jahrestagung des Südosteuropäischen Germanistenverbandes, Kragujevac, 11.-15. November 2015. Kragujevac: Filum, S. $55-68$.

/51/ Maz. Wb. (2005). Толковен речник на македонскиот јазик, том II, 3-K. Составувачи под раководство на Снежана Велковска. Скопје: Институт за македонски јазик „Крсте Мисирков“.

[Maz. Wb. (2005). Tolkoven rečnik na makedonskiot jazik, tom II, Z-K. Sostavuvači pod rakovodstvo na Snežana Velkovska. 
Skopje: Institut za makedonski jazik „Krste Misirkov“.]

/52/ Maz. Wb. (2014). Толковен речник на македонскиот јазик, том VI, Т-Ш. Составувачи под раководство на Снежана Велковска. Скопје: Институт за македонски јазик „Крсте Мисирков“.

[Maz. Wb. (2014). Tolkoven rečnik na makedonskiot jazik, tom VI, T- Š. Sostavuvači pod rakovodstvo na Snežana Velkovska. Skopje: Institut za makedonski jazik „Krste Misirkov".]

/53/ Gehl, H. (2003). Wörterbuch der donauschwäbischen Landwirtschaft. Stuttgart: Steiner.

/54/ Grčeva, R. und Rau, P. (2006). Голем македонскогермански и германско-македонски речник / Großes mazeonisch-deutsches und deutschmazedoniches Wörterbuch. Скопје: Магор / Skopje: Magor.

/55/ Schleiermacher, F. (1963 [1918]). Ueber die verschiedenen Methoden des Uebersezens. In: H. J. Störig (Hg.): Das Problem des Übersetzens. Darmstadt: Wissenschaftliche Buchgesellschaft, 38-70 (file:///C:/Users/user/Documents/Translation/Sch leiermacher.pdf; Zugriff: 28.4.2018).
/56/ Kučiš, Vlasta (2017). Eine Reise durch die Geschichte der Translation. In: Kučiš, Vlasta (Hrg.), Žagar-Šoštarić, Petra (Hrg.). Beiträge zur Translation von gestern, heute und morgen. Rijeka: Filozofski fakultet Sveučilišta. 2017, 12-28 (http://izdavastvo.ffri.hr/wp-content/ uploads/2017/01/END_WEB_VERSION.pdf (23.4.2018)

/57/ Ibid.

Literatur

Originalwerke

Конески, Б. (1967). Љубов, Лозје. Скопје: Култура (1955),

62-85.

[Koneski, B. (1967). Ljubov, Lozje. Skopje: Kultura (1955), 62-85.]

Конески, Б. (1967). Песна, Лозје. Скопје: Култура (1955), 41-46.

[Koneski, B. (1967). Pesna, Lozje. Skopje: Kultura (1955),

41-46.]

Übersetzungen

Koneski, B. (1967). Liebe. In: J. Manfred (Hrsg.): Jugoslawische Erzähler von Lazarević bis Andrić. Aus dem Mazedonischen: K. Gutschmidt. Leipzig: Dieterich, S. 511-516.

Koneski, B. (1972): „Petres Lied“. In: Jugoslawische Erzählungen. Aus dem Mazedonischen von A. Philippsen. Leipzig: Philipp, 302-307 